\title{
COMPARATIVE PHARMACOKINETICS OF RHUBARB ANTHRAQUINONES LOADED NANOEMULSION BY DIFFERENT PLASMA DRUG CONCENTRATION CALCULATION METHODS
}

\author{
GUIFANG ZHANG ${ }^{1}$, JINCHENG LI ${ }^{1}$, HUIJUAN LV ${ }^{1}$, CHUNXI QIAN ${ }^{1}$, XUEFENG LI², \\ ZHIGANG YANG ${ }^{1}$, JIANYIN LI' ${ }^{1}$, SHUAIBO YANG ${ }^{1}$, BANDAR MOHAMMED AL HAMYARI', \\ and YANBIN SHI ${ }^{*}$
}

'School of Pharmacy, Lanzhou University, Lanzhou 730000, China

${ }^{2}$ School of Basic Medical Sciences, Lanzhou University, Lanzhou 730000, China

\begin{abstract}
Rhubarb is widely used to treat constipation, hyperlipidemia, and obesity in China and other Asian countries. Rhubarb free anthraquinones (RhA) are the major components of rhubarb, which are considered to be responsible for its therapeutic effect. In this paper, RhA was constructed into RhA loaded nanoemulsion to increase their solubility and bioavailability. The contents of RhA monomers (aloe-emodin, rhein, emodin, chrysophanol, and physcion) in rat plasma were determined by HPLC equipped with YMC-Pack-ODS-C18 column. The mobile phase was selected as methanol: $0.1 \%$ phosphoric acid $(75: 25, \mathrm{v} / \mathrm{v})$ with isocratic elution. Fluorescence detection was employed with an excitation wavelength of $435 \mathrm{~nm}$ and an emission wavelength of $515 \mathrm{~nm}$. The total plasma RhA concentrations were individually calculated by adding-up and integration methods. The pharmacokinetic parameters were obtained by statistical moment analysis. The values of concentration-related parameters obtained by the adding-up method were higher than those obtained by the integration method, while the values of time-related pharmacokinetic parameters are in an indistinctive range. The results provided factual evidence for the application of integrated pharmacokinetics with the integrated plasma drug concentration calculation method.
\end{abstract}

Keywords: Rhubarb anthraquinones, Nanoemulsion, HPLC-FLD, Integrated pharmacokinetics.

Rhubarb is the dried root and rhizome of Rheum Palmatum L., Rheum tanguticum Maxim. ex Balf. or Rheum officinale Baill. distributed in western China and some Asian countries. It is one of the most widely-used traditional Chinese medicine (abbreviated as TCM). Rhubarb has remarkable purging and heat-clearing effects according to the unique theory of TCM. Modern studies have confirmed that rhubarb has various pharmacological activities such as catharsis (1), anti-inflammation (2), anti-bacteria (3), anti-virus (4), anti-oxidation (5), liver protection (6), anti-tumor (7), and anti-constipation (8). Anticonstipation action was confirmed partly caused by rhubarb anthraquinones, which was achieved by inhibiting the expression of aquaporin in the intestinal epithelial cell membrane, and the inhibition rate depended on the concentration of anthraquinones distributed in the intestine but not absorbed into the blood $(8,9)$. Therefore, we hypothesized the lower plasma concentration of the total rhubarb anthraquinones is more consistent with the situation that only non-absorbed rhubarb anthraquinone produced the purgative effect. Generally, the plasma concentration-related pharmacokinetic parameters $\left(\mathrm{AUC}_{0-\infty}\right.$ and $\mathrm{C}_{\text {max }}$ ) are adopted to evaluate the absorption levels of active components.

The main chemical components of rhubarb include anthraquinones, tannins, and polysaccharides. Anthraquinones include free forms (abbreviated as $\mathrm{RhA}$ ) and bound forms with saccharides. RhA mainly includes aloe-emodin, rhein, emodin, chrysophanol, and physcion (Figure 1) (10). These compounds

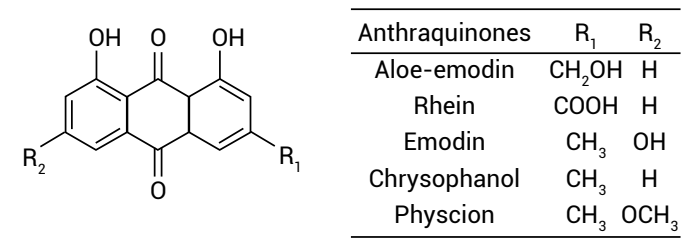

Figure 1. The chemical structure of rhubarb free anthraquinones.

* Corresponding author: e-mail: shiyb@1zu.edu.cn 
are difficult to be dissolved in water. After oral administration, aloe-emodin, emodin, chrysophanol, and physcion are prone to form glucuronides, resulting in poor oral absorption and low bioavailability.

Nanoemulsion is a heterogeneous liquid dispersion system with a particle size of 10-100 nm formed by immiscible two-phase liquid under emulsification. Good kinetic stability, low viscosity, and high optical transparency make it a very attractive drug delivery system for industrial applications $(11,12)$. If RhA were encapsulated in $\mathrm{O} / \mathrm{W}$ nanoemulsion, their solubility in water would increase. It is even more useful, that RhA can be avoided from being metabolized in the intestinal tract and easily penetrated by the intestinal mucosal. As a whole, their oral bioavailability can be significantly improved $(10,13)$.

Pharmacokinetic parameters such as the area under drug concentration-time curve (AUC), maximum plasma drug concentration $\left(\mathrm{C}_{\max }\right)$, and its corresponding time $\left(\mathrm{t}_{\max }\right)$ are generally used to evaluate bioavailability. Some studies have adopted multiple components of TCM to investigate their pharmacokinetics and bioavailability but fail to reflect the overall synergistic effect among different components (1). Currently, literature on pharmacokinetics concerning RhA mainly focuses on a single component and its drug delivery system $(10,13)$. Wu et. al. reported that the plasma drug concentrations of all five RhA monomers were determined simultaneously, but their pharmacokinetic parameters were separately analyzed based on their respective plasma concentration $(14,15)$.

In our previous study, the plasma concentration of each RhA monomer was applied to analyze their respective pharmacokinetics of RhA loaded nanoemulsion (RhA-NE) in rats by single oral administration, and the overall pharmacokinetics were also analyzed using the total plasma RhA concentration calculated with a simple mathematical adding-up method (10). Briefly, the adding-up concentration calculation method is that the plasma concentrations of five RhA monomers at the same sampling time point were summed up without weight coefficient, and then the overall pharmacokinetic parameters were calculated based on the adding-up plasma concentrations. However, the plasma concentrationrelated pharmacokinetic parameters $\left(\mathrm{AUC}_{0-\infty}\right.$ and $\mathrm{C}_{\text {max }}$ ) were higher than we expected. That means the synergic effect of natural compounds may not be reflected properly by the adding-up method. These pharmacokinetic parameters could not objectively reflect the integrated pharmacokinetic behavior of RhA-NE, because the bioavailability of each RhA monomer was different after oral administration.
In recent years, integrated pharmacokinetics was proved to be more suitable for multiple components and multiple-targeted medicine, which can explain their pharmacological effects more reasonably and comprehensively $(16,17)$.

In this paper, after RhA-NE was orally administered by rats, plasma concentrations of five RhA monomers were determined by high-performance liquid chromatography fluorescence detection (HPLC-FLD). The total plasma concentrations of RhA were individually calculated by adding-up and integration methods. The pharmacokinetic parameters were achieved by statistical moment analysis. Finally, the differences in the integrated pharmacokinetic properties obtained by the two plasma RhA concentration calculation methods were compared. The results presented practical data to demonstrate the pharmacokinetic difference of multiple components with different plasma drug concentration calculation methods, providing factual evidence for the application of integrated pharmacokinetics with the integrated plasma drug concentration calculation method.

\section{MATERIALS AND METHODS}

\section{Chemicals and materials}

The dried radix et rhizoma of Rheum palmatum L. was purchased from the Lixian Pharmaceutical Company, Gansu province of China, and identified by professor Yongjian Yang working in Lanzhou University. A voucher specimen (No. 856002) was deposited in the Institute of Pharmacognosy, School of Pharmacy of Lanzhou University. Reference standards of aloe-emodin, rhein, emodin, chrysophanol, and physcion, as well as 1,8-dihydroxyanthraquinone solution (internal standard), were purchased from the China Institute for the Control of Pharmaceutical and Biological Products. Cremophor RH 40 was purchased from BASF company (Germany). Capryol 90 and Transcutol HP were donated by Gattefosse (Shanghai) Trading Co., Ltd. Methanol (HPLC grade) was obtained from Fisher Scientific Ltd. (Pittsburgh, USA).

\section{Preparation of RhA-NE}

RhA-NE was prepared by titration method according to the formulation optimization reported by our research group (10). Briefly, RhA (5.1 mg) was accurately weighed and added into $1.0 \mathrm{~mL}$ mixed oil composed of capryol 90 and ethyl oleate $(3: 1$, $\mathrm{W} / \mathrm{W}$ ), which accounted for $19.5 \%$ of the total nanoemulsion. $1.0 \%$ oleic acid as a stabilizer was added to the mixed oil. Cremophor RH 40 as a surfactant 
was heated and melted at $35^{\circ} \mathrm{C}$, and then transcutol $\mathrm{HP}$ as co-surfactant was added and stirred evenly to obtain a mixed emulsifier $(1: 1, \mathrm{~W} / \mathrm{W})$, which accounted for $42.5 \%$ of the total nanoemulsion. The mixed emulsifier was added into the oil phase, stirred until it became homogeneous. Finally, deionized water as an aqueous phase accounted for $36.5 \%$ of the total nanoemulsion, was added dropwise under continuous stirring to form transparent $\mathrm{O} / \mathrm{W}$ nanoemulsion.

\section{Pretreatment of plasma samples}

A plasma sample of $1.0 \mathrm{~mL}$ was taken and placed in a centrifuge tube. $200 \mu \mathrm{L}$ of chromatographic grade methanol as protein precipitating reagent and solvent, $200 \mu \mathrm{L}$ of hydrochloric acid solution $(2.0 \mathrm{~mol} / \mathrm{L})$, and $100 \mu \mathrm{L}$ of 1,8 -dihydroxyanthraquinone solution (internal standard, IS, $128 \mathrm{ng} / \mathrm{mL}$ ) were added into the above plasma sample. They were mixed on a vortex mixer for $1 \mathrm{~min}$, centrifuged at $5000 \mathrm{rpm}$. The supernatant was added with $4.0 \mathrm{~mL}$ ethyl acetate to extract target compounds. The organic layer was concentrated and dried at $40^{\circ} \mathrm{C}$ with a rotary evaporator under reduced pressure. The residue was re-dissolved with $200 \mathrm{~mL}$ methanol, filtered with a $0.45 \mu \mathrm{m}$ organic membrane (Shanghai Xingya purification material factory), and the filtrate was injected into HPLC for plasma concentration analysis.

\section{Standard and quality control sample preparation}

Serial standard working solutions of RhA monomers were prepared with methanol as solvent. Standard curve samples were prepared by adding proper standard working solutions into $1.0 \mathrm{~mL}$ blank plasma to predetermined concentrations. The detailed concentrations were different for each RhA monomer due to their different contents in RhA. Thus, lower, medium and higher concentrations of quality control (QC) samples were also different for each monomer. The preparation process was the same as Section Pretreatment of plasma samples. The peak area ratio (y) of RhA and IS was used as the ordinate, and the plasma drug concentration ( $\mathrm{x}$, $\mathrm{ng} / \mathrm{mL}$ ) was used as the abscissa to draw the graph and fit the standard curve.

\section{HPLC analysis}

HPLC (Agilent 1260) was equipped with G1311B quaternary pump, G1316A column thermostat, G1329B autosampler, G1315D diode array detector. Chromatographic separation was achieved on a YMC-Pack-ODS-C18 chromatographic column $(150 \mathrm{~mm} \times 4.6 \mathrm{~mm}, 5 \mu \mathrm{m})$ preceded by a SecurityGuard C18 pre-column (Phenomenex, USA). The mobile phase was selected as methanol- $0.1 \%$ phosphoric acid $(75: 25, \mathrm{~V} / \mathrm{V})$ at a flow rate of $0.8 \mathrm{~mL} / \mathrm{min}$. Fluorescence detection was employed to monitor data with an excitation wavelength of $435 \mathrm{~nm}$ and an emission wavelength of $515 \mathrm{~nm}$. The column temperature was maintained at $40^{\circ} \mathrm{C}$. The injection volume of all samples was $20 \mu \mathrm{L}$. Specificity, linearity, LOD and LOQ, precision, accuracy, and recovery at three levels were investigated. The LOD and LOQ were calculated by the formula: $\mathrm{LOD}=3 \times \mathrm{SD} \times \mathrm{C} / \mathrm{A} ; \mathrm{LOQ}=10 \times \mathrm{SD} \times \mathrm{C} / \mathrm{A}$. where 3 and 10 were the values of signal-to-noise $(\mathrm{S} / \mathrm{N})$ ratio for $\mathrm{LOD}$ and $\mathrm{LOQ}$, respectively; SD is standard deviation obtained from ten analysis results of a low concentration of analytes; $\mathrm{C}$ is the concentration of the analytes; $\mathrm{A}$ is the average HPLC response of more than six analyses. The accuracy was evaluated by the relative recovery of the analytes, which was calculated by the response value of the target substance recovered from the biological sample matrix divided by the response value of the standard substance added to the biological matrix in known quantities. The extraction recovery of the analyte is the response value of the target substance recovered from the matrix of the biological sample divided by the response value generated by the pure standard substance. The stability tests included: (1) The spiked plasma samples at three levels were stored at $25^{\circ} \mathrm{C}$ for $24 \mathrm{~h}$; (2) The spiked plasma samples at three levels were stored at $-20^{\circ} \mathrm{C}$ for 5, 15 and 30 days; (3) The spiked plasma samples at three levels undergone three freeze-thaw cycles $\left(-20^{\circ} \mathrm{C}\right.$ to ambient temperature) with each cycle of $24 \mathrm{~h}$. The RhA concentration change rate (n,\%) of plasma sample concentration was measured according to the formula (1).

$$
\mathrm{n}(\%)=\frac{C_{0}-C_{\mathrm{i}}}{C_{0}} \times 100 \%
$$

In the formula, $\mathrm{C}_{0}$ represents the initial plasma concentration of RhA monomers; $\mathrm{C}_{\mathrm{i}}$ represents the measured plasma concentration of RhA monomers at different times.

\section{Pharmacokinetic experiments}

Wistar male rats of $240 \pm 20 \mathrm{~g}$ were supplied by the Experimental Animal Center of Lanzhou University (Lanzhou, China). The Guidelines for the Care and Use of Laboratory Animals were abided according to the US National Research Council (1996) and the related ethic regulations of Lanzhou University during the whole animal experiment. Rats 
were acclimated in a temperature-controlled breeding room for at least one week before starting the experiments. Prior to administration, the rats fasted for $12 \mathrm{~h}$ with water ad libitum.

Rats were administered RhA-NE by gavage at a single dose of $15 \mathrm{mg} / \mathrm{Kg}$. After $4 \mathrm{~h}$ of gavage, the rats were free to eat and drink. Blood (about $2.5 \mathrm{~mL}$ for each rat) was drawn from the heart at $0.25,0.5,1,1.5,2,4,6,8,12,18,24,48 \mathrm{~h}$ after the rats were anesthetized. Three blood samples were respectively collected from three rats at each time point and transferred into heparinized tubes, centrifuged at $12000 \mathrm{rpm}$ for $10 \mathrm{~min}$ to obtain plasma samples which were stored at $-20^{\circ} \mathrm{C}$ until pretreatment and analysis. Each plasma sample was analyzed by HPLC-FLD in duplicate. The total plasma RhA concentration was separately calculated by the adding-up and the integration method. The adding-up method is that the plasma concentration of the total RhA was obtained by directly summing up the plasma concentration of each monomer without weight coefficient at each time point. The integration method is about summing up the area under the concentration-time curve $\left(\mathrm{AUC}_{0-\infty}\right)$ of each monomer, then calculating the ratio of the AUC of each monomer to the total RhA as weight coefficients ( $\omega \mathrm{j}$, formula 2 ). The total plasma RhA concentrations (Cint) were obtained by calculating the weighted sum of the plasma concentration of different monomers at each time point (formulas 2-3) (17). The pharmacokinetic parameters were further analyzed with the total plasma RhA concentration calculated by different methods using the pharmacokinetic program of DAS 3.2.7 edited by Drug and Statistics, Mathematical Pharmacology Professional Committee of China.

$$
\begin{gathered}
\omega_{j}=\frac{A U C_{j 0-\infty}}{\sum_{1}^{5} A U C_{0-\infty}} \\
\text { Cint }=\omega_{1} \times C_{1}+\omega_{2} \times C_{2}+\omega_{3} \times C_{3}+ \\
+\omega_{4} \times C_{4}+\omega_{5} \times C_{5}
\end{gathered}
$$

In the formula, $\omega$ represents the weight coefficient; $\mathrm{j}$ represents five RhA monomers; $\mathrm{C}$ represents the concentration of each RhA monomer; Cint represents the total plasma RhA concentration.

\section{RESULTS}

\section{Method validation}

Typical chromatograms of rat plasma samples are shown in Figure 2. The retention time of aloe-emodin, rhein, IS, emodin, chrysophanol, and physcion
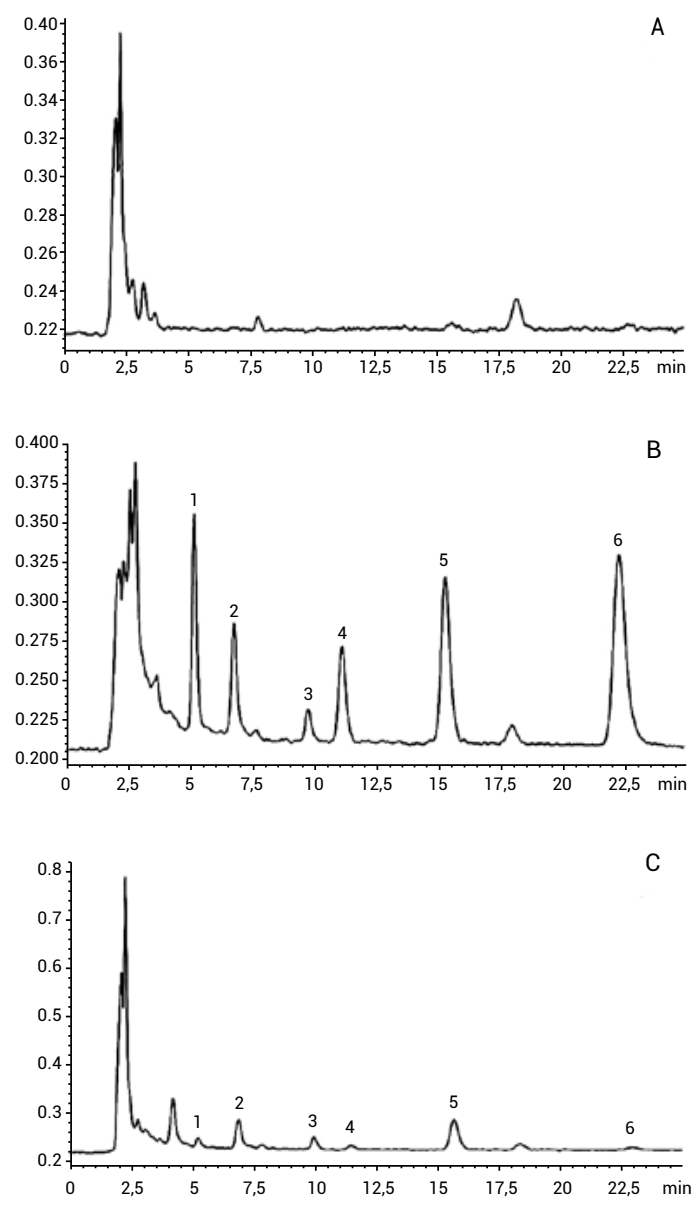

Figure 2. Typical chromatograms of rat plasma samples (A) blank plasma; (B) plasma sample spiked with mixed standards; (C) plasma samples after oral administration of RhA loaded nanoemulsion. Peaks 1, 2, 3, 4, 5, and 6 respectively represent aloe-emodin, rhein, IS, emodin, chrysophanol, physcion.

were respectively $5.28 \pm 0.25 \mathrm{~min}, 7.15 \pm 0.32 \mathrm{~min}$, $10.19 \pm 0.38 \mathrm{~min}, 11.55 \pm 0.45 \mathrm{~min}, 15.74 \pm 0.56 \mathrm{~min}$, and $23.28 \pm 0.68 \mathrm{~min}$, indicating all tested samples can achieve baseline separation under the chromatographic condition. There were no endogenous peaks significantly interfering with the analytes. The correlation coefficients of the standard curve of plasma RhA monomers, LOD and LOQ are listed in Table 1. The precision, accuracy, and recovery of RhA monomers at three levels are listed in Table 2. The RhA concentration change rate $(\mathrm{n}, \%)$ of plasma samples of the stability tests at different conditions are shown in Table 3.

\section{Pharmacokinetics}

The plasm concentrations at different sampling time points were calculated according to the standard curves of each RhA monomer. The mean plasma concentrations of five RhA monomers v.s. time profiles 
Table 1. Standard curves, linear range, LOD, and LOQ.

\begin{tabular}{|c|c|c|c|c|c|}
\hline Analytes & Fitted equation & $\begin{array}{c}\text { Correlation } \\
\text { coefficient }\end{array}$ & Linear range $(\mathrm{ng} / \mathrm{mL})$ & LOD (ng/mL) & LOQ (ng/mL) \\
\hline Aloe emodin & $y=24064.18 x+0.03768$ & 0.9998 & $4.53-507.82$ & 1.21 & 4.04 \\
\hline Rhein & $y=14367.22 x-0.00383$ & 0.9987 & $14.90-1097.03$ & 3.74 & 12.47 \\
\hline Emodin & $y=15065.34 x+0.08961$ & 0.9989 & $13.62-580.07$ & 3.58 & 11.93 \\
\hline Chrysophanol & $y=30925.68 x+0.03694$ & 0.9997 & $5.98-2246.09$ & 1.29 & 4.30 \\
\hline Physcion & $y=52055.05 x+0.10439$ & 0.9996 & $5.77-218.52$ & 1.40 & 4.78 \\
\hline
\end{tabular}

Table 2. Intra- and inter-day precision, accuracy, and recovery of the RhA monomers in rat plasma.

\begin{tabular}{|c|c|c|c|c|c|}
\hline Analytes & $\begin{array}{l}\text { Conc. } \\
(\mathrm{ng} / \mathrm{mL})\end{array}$ & $\begin{array}{c}\text { Intra-day precision } \\
(\%)\end{array}$ & $\begin{array}{c}\text { Inter-day precision } \\
(\%)\end{array}$ & $\begin{array}{l}\text { Accuracy } \\
(\%)\end{array}$ & $\begin{array}{c}\text { Extraction recovery } \\
(\%)\end{array}$ \\
\hline \multirow{3}{*}{ Aloe emodin } & 504.28 & 2.07 & 2.42 & 101.1 & 98.3 \\
\hline & 51.07 & 4.35 & 5.53 & 95.8 & 92.6 \\
\hline & 12.77 & 5.09 & 6.32 & 102.2 & 95.2 \\
\hline \multirow{3}{*}{ Rhein } & 1080.40 & 2.62 & 3.66 & 102.6 & 96.7 \\
\hline & 216.08 & 5.82 & 6.24 & 102.3 & 95.2 \\
\hline & 27.01 & 6.69 & 8.86 & 104.5 & 95.6 \\
\hline \multirow{3}{*}{ Emodin } & 575.90 & 3.24 & 4.68 & 100.3 & 92.2 \\
\hline & 115.18 & 3.62 & 5.12 & 95.8 & 90.8 \\
\hline & 28.80 & 7.15 & 8.62 & 90.2 & 85.2 \\
\hline \multirow{3}{*}{ Chrysophanol } & 2120.10 & 2.02 & 3.65 & 99.7 & 96.8 \\
\hline & 112.01 & 3.52 & 4.56 & 99.2 & 91.5 \\
\hline & 14.00 & 4.26 & 7.82 & 106.5 & 96.2 \\
\hline \multirow{3}{*}{ Physcion } & 220.48 & 3.42 & 4.65 & 99.5 & 97.5 \\
\hline & 55.12 & 4.32 & 6.23 & 92.0 & 89.8 \\
\hline & 13.78 & 5.85 & 8.55 & 102.4 & 89.6 \\
\hline
\end{tabular}

Table 3. Stability results of the RhA monomers in plasma.

\begin{tabular}{|c|c|c|c|c|c|c|c|c|}
\hline \multirow{3}{*}{ Analytes } & \multirow{3}{*}{$\begin{array}{l}\text { Conc. } \\
\text { (ng/mL) }\end{array}$} & \multicolumn{7}{|c|}{$\mathrm{n}(\%)$} \\
\hline & & \multirow{2}{*}{$\frac{\text { Stability at } 25^{\circ} \mathrm{C}}{24 \mathrm{~h}}$} & \multicolumn{3}{|c|}{ Stability at $-20^{\circ} \mathrm{C}$} & \multicolumn{3}{|c|}{ Freeze-thaw stability } \\
\hline & & & $5 d$ & $15 \mathrm{~d}$ & $30 \mathrm{~d}$ & $1 d$ & $2 d$ & $3 d$ \\
\hline \multirow{3}{*}{ Aloe emodin } & 504.28 & 2.67 & 1.53 & 2.06 & 2.22 & 1.61 & 2.17 & 3.35 \\
\hline & 51.07 & 4.34 & 1.27 & 1.39 & 3.36 & 1.28 & 3.62 & 5.36 \\
\hline & 12.77 & 5.25 & -0.38 & 2.84 & 4.65 & -0.34 & 3.81 & 6.96 \\
\hline \multirow{3}{*}{ Rhein } & 1080.40 & 3.52 & 1.65 & 2.96 & 3.21 & 1.47 & 2.06 & 4.45 \\
\hline & 216.08 & 5.95 & 1.33 & 2.87 & 3.86 & 1.16 & 3.61 & 6.86 \\
\hline & 27.01 & 7.26 & -0.24 & 2.68 & 4.85 & -0.18 & 4.39 & 7.82 \\
\hline \multirow{3}{*}{ Emodin } & 575.90 & 3.45 & 1.36 & 2.06 & 3.07 & 1.43 & 3.52 & 5.47 \\
\hline & 115.18 & 5.69 & 1.92 & 3.28 & 4.32 & 2.25 & 4.67 & 7.49 \\
\hline & 28.80 & 7.47 & 2.16 & 3.65 & 5.26 & 2.08 & 5.86 & 8.34 \\
\hline \multirow{3}{*}{ Chrysophanol } & 2120.10 & 3.52 & 0.58 & 1.35 & 2.82 & 1.66 & 2.41 & 4.38 \\
\hline & 112.01 & 5.54 & 1.26 & 2.82 & 3.88 & 1.52 & 3.58 & 5.65 \\
\hline & 14.00 & 6.25 & 0.49 & 2.93 & 4.22 & -0.48 & 4.72 & 7.63 \\
\hline \multirow{3}{*}{ Physcion } & 220.48 & 3.56 & 1.91 & 2.06 & 2.86 & 1.64 & 2.43 & 4.12 \\
\hline & 55.12 & 5.28 & 0.82 & 1.85 & 3.12 & 2.15 & 4.08 & 6.37 \\
\hline & 13.78 & 6.52 & -0.27 & 2.73 & 4.69 & 0.47 & 5.91 & 7.85 \\
\hline
\end{tabular}



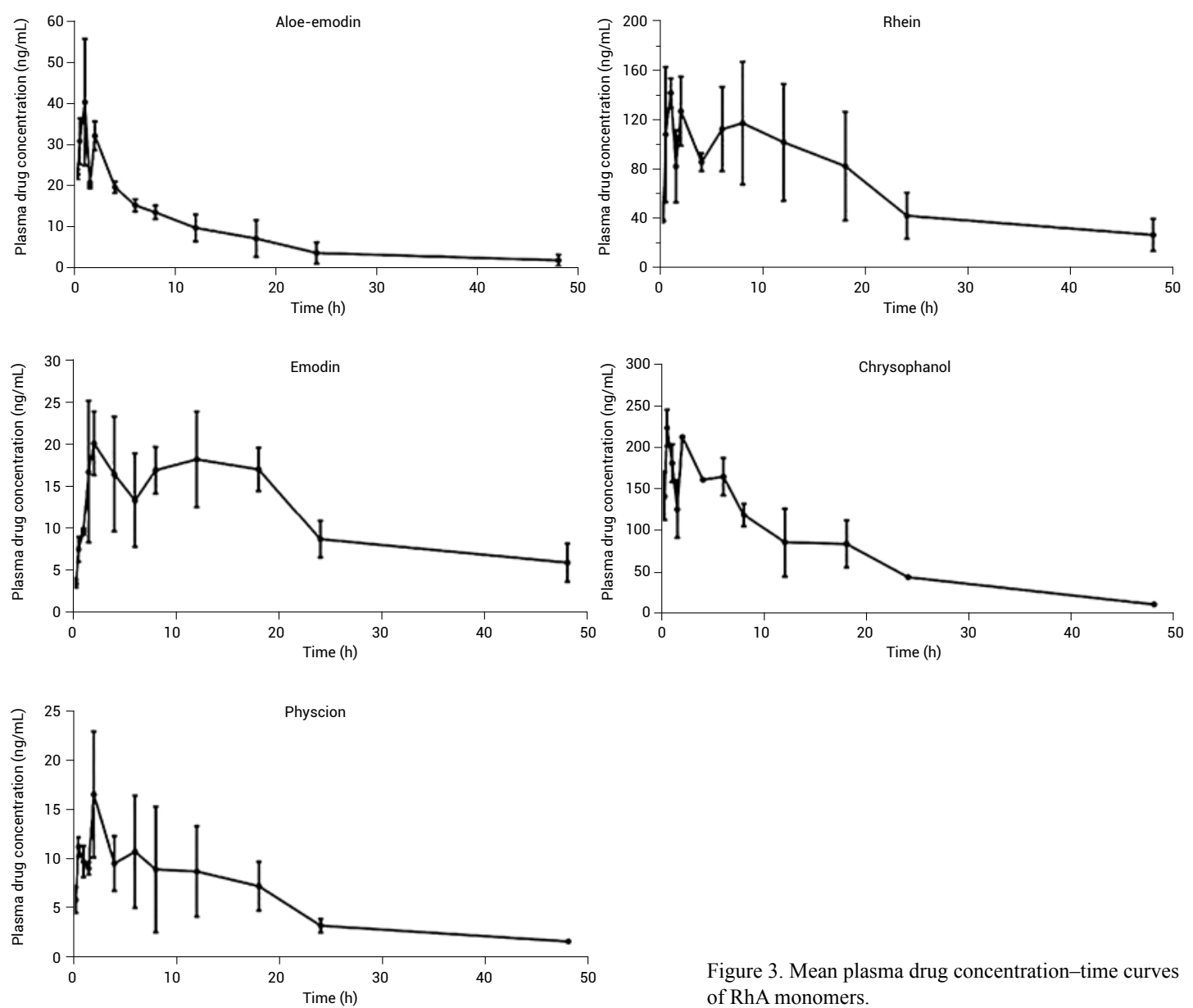

Figure 3. Mean plasma drug concentration-time curves of RhA monomers.

are shown in Figure 3. The profiles demonstrate two successive maximum concentration peaks for all of the five RhA monomers. Statistical moment model was chosen to analyze pharmacokinetic parameters. The pharmacokinetic parameters of each RhA monomer were shown in Table 4. In order to better understand the holistic pharmacokinetics of RhA-NE, the

total plasma RhA concentrations at different sampling time points were individually calculated by adding-up and integration methods. The corresponding total plasma RhA concentration-time curves were separately shown in Figure 4. The pharmacokinetic parameters obtained by the two plasma drug concentration calculation methods are listed in Table 4.
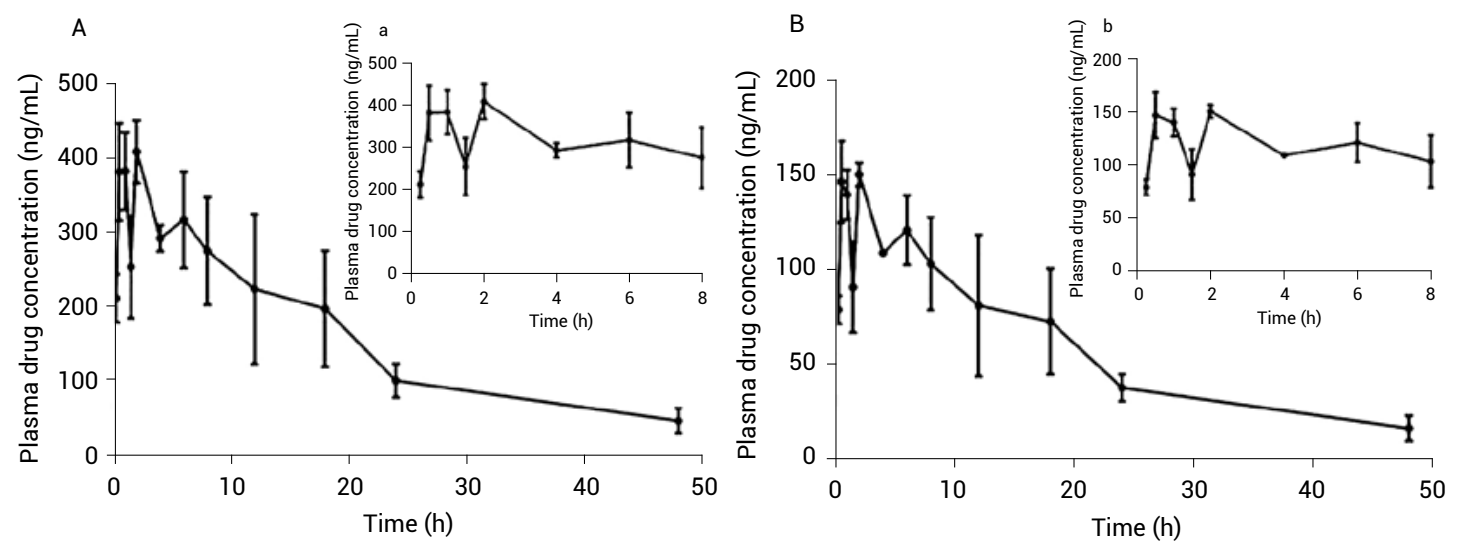

Figure 4. Plasma drug concentration-time curves of the total RhA (A) adding-up method (0-48 h), (a) adding-up method (0-8 h); and $(\mathrm{B})$ integration method $(0-48 \mathrm{~h})$, (b) integration method $(0-8 \mathrm{~h})$ 


\section{DISCUSSION AND CONCLUSION}

Rhubarb-free anthraquinones are water-insoluble compounds. Relatively, they have good solubility in neutral to alkaline water-based liquid because part of them exist in ionic form. However, RhA in the ionic form is difficult to be extracted by ethyl acetate, so hydrochloric acid was added to adjust the $\mathrm{pH}$ of plasma to be more acidic to ensure RhA exists in molecular form. While the solubility of RhA would be reduced in acidic plasma, and small volume of methanol was added to prevent RhA from precipitation. Although the added volume of methanol is small, it can precipitate small amounts of protein. Therefore, the role of methanol was acted as protein precipitating reagent and solvent.

The method validation data (Table 1-3) substantiated that the HPLC analysis and sample processing were considered generally meeting the requirements of biological sample analyses. However, since the plasma samples at each time point were from three rats, the error of plasma drug concentration was large (Figure 3 and Figure 4), and the error mainly came from individual differences of rats and sample treatment processes.

Considering lower limit of detection (LOD) generated from a larger volume of plasma sample benefits the achievement of more valuable pharmacokinetic parameters, three blood samples were respectively collected from three rats at each time point, and about $2.5 \mathrm{~mL}$ blood for each rat was drawn from the heart after the rats were anesthetized. The rats were sacrificed and the tissues were collected for future drug distribution analyses.

The double peaks in plasma drug concentration-time curves indicated the absorption and disposition of RhA in vivo are complex. Physiological processes, enterohepatic recycling, and multiple absorption sites are often associated with multiple peak occurrences in pharmacokinetic studies (18, 19). It can be seen from Figure 4. that the plasma drug concentration-time curves obtained by two concentration calculation methods are similar in shape, and the bimodal phenomenon occurs in the same period of sampling time. In fact, the bimodal phenomena of the above curves are also observed in the pharmacokinetic profiles of RhA monomers (Figure 3), indicating the holistic pharmacokinetic characteristics of the total $\mathrm{RhA}$ reflected that of each RhA monomer.

\begin{tabular}{|c|c|c|c|c|c|c|c|c|c|}
\hline 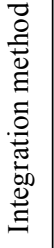 & 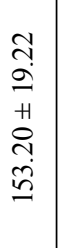 & 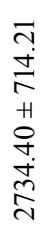 & 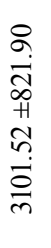 & $\begin{array}{l}\stackrel{2}{\hat{0}} \\
\text { H } \\
+ \\
+ \\
\dot{ \pm}\end{array}$ & 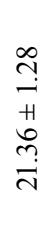 & \begin{tabular}{l}
$\infty$ \\
$\infty$ \\
0 \\
+ \\
0 \\
0 \\
\hdashline
\end{tabular} & $\begin{array}{l}+ \\
\infty \\
0 \\
0 \\
H \\
0 \\
\infty \\
0 \\
0\end{array}$ & $\begin{array}{l}\vec{\infty} \\
0 \\
\dot{m} \\
+ \\
0 \\
\infty \\
\stackrel{j}{ \pm} \\
=\end{array}$ & 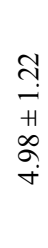 \\
\hline 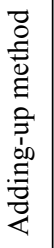 & 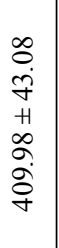 & 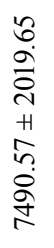 & 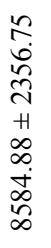 & $\begin{array}{l}\text { مे } \\
0 \\
H \\
0 \\
\ddot{n} \\
\qquad\end{array}$ & 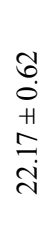 & $\begin{array}{l}\infty \\
\infty \\
0 \\
+1 \\
0 \\
\stackrel{n}{-}\end{array}$ & $\begin{array}{l}\tilde{n} \\
\stackrel{3}{-} \\
+1 \\
0 \\
\tilde{n} \\
0\end{array}$ & 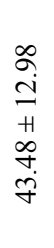 & $\begin{array}{l}\underset{+}{\sigma} \\
\dot{0} \\
+ \\
+ \\
+ \\
-\end{array}$ \\
\hline 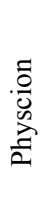 & $\begin{array}{l}8 \\
0 \\
+ \\
+1 \\
\hat{\sigma} \\
\sigma\end{array}$ & 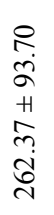 & 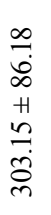 & 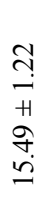 & $\begin{array}{l}\bar{\sigma} \\
\dot{+} \\
+1 \\
\infty \\
\infty \\
\dot{j}\end{array}$ & $\begin{array}{l}\infty \\
0 \\
0 \\
+1 \\
0 \\
0 \\
-1\end{array}$ & 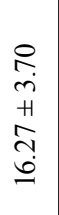 & 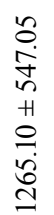 & $\begin{array}{l}n \\
n \\
n \\
H \\
\text { on } \\
\text { in } \\
n\end{array}$ \\
\hline 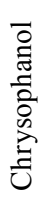 & 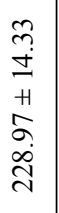 & 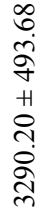 & 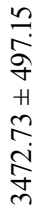 & $\begin{array}{l}\text { oे } \\
0 \\
+ \\
\infty \\
\sim \\
\end{array}$ & $\begin{array}{l}\infty \\
n \\
0 \\
+ \\
+ \\
\hat{a} \\
\vdots \\
n\end{array}$ & $\begin{array}{l}0 \\
0 \\
0 \\
H \\
0 \\
0 \\
0\end{array}$ & $\begin{array}{l}0 \\
0 \\
0 \\
+1 \\
0 \\
0 \\
\dot{\Xi}\end{array}$ & $\begin{array}{l}n \\
\stackrel{+}{ \pm} \\
+1 \\
\infty \\
0 \\
\stackrel{8}{8}\end{array}$ & $\begin{array}{l}\tilde{n} \\
0 \\
+ \\
\infty \\
\infty \\
\sim \\
+\end{array}$ \\
\hline 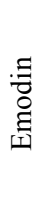 & 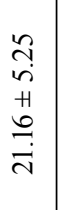 & $\begin{array}{l}m \\
\infty \\
\infty \\
\cong \\
+ \\
\infty \\
\infty \\
\infty \\
\infty \\
\dot{\infty}\end{array}$ & 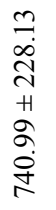 & $\begin{array}{l}n \\
\hat{0} \\
+1 \\
0 \\
\tilde{n} \\
\infty\end{array}$ & $\begin{array}{l}n \\
0 \\
\infty \\
H \\
n \\
\infty \\
\infty \\
m\end{array}$ & $\begin{array}{l}\stackrel{2}{\tilde{y}} \\
0 \\
+ \\
\tilde{\infty} \\
\stackrel{\infty}{-}\end{array}$ & 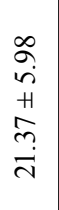 & 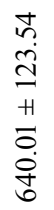 & 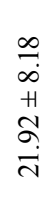 \\
\hline$\frac{\Xi}{\mathbb{E}}$ & 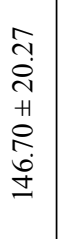 & 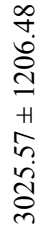 & 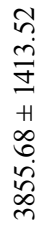 & 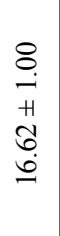 & $\begin{array}{l}\hat{o} \\
\stackrel{a}{0} \\
+ \\
\stackrel{n}{\rho} \\
\stackrel{m}{m}\end{array}$ & $\begin{array}{l}\text { సे } \\
0 \\
+ \\
\tilde{0} \\
\infty \\
0\end{array}$ & $\begin{array}{l}\hat{b} \\
a \\
+ \\
\hat{a} \\
\hat{a} \\
\tilde{N}\end{array}$ & 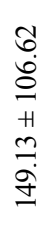 & $\begin{array}{l}\stackrel{+}{+} \\
\stackrel{+}{H} \\
\tilde{\sim} \\
\stackrel{+}{+}\end{array}$ \\
\hline 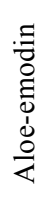 & $\begin{array}{l}\stackrel{0}{1} \\
0 \\
+ \\
+1 \\
\check{c} \\
\dot{+}\end{array}$ & 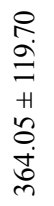 & 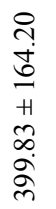 & $\begin{array}{l}\stackrel{0}{n} \\
i \\
H \\
\stackrel{n}{n} \\
\stackrel{y}{=}\end{array}$ & 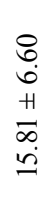 & $\begin{array}{l}\text { సे } \\
0 \\
H \\
\tilde{H} \\
0 \\
0\end{array}$ & 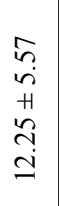 & 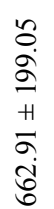 & 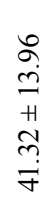 \\
\hline & 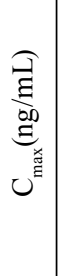 & 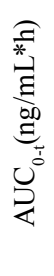 & 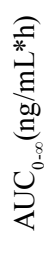 & 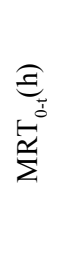 & 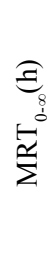 & 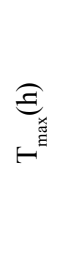 & $\underset{\mathbb{N}}{\underbrace{}_{\mathbb{N}}}$ & 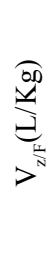 & 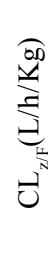 \\
\hline
\end{tabular}


The total plasma RhA concentration obtained by the adding-up method was about 3 times higher than that obtained by the integration method. This was because the adding-up method was equivalent to assigning a weight coefficient of 1 to the concentration of each RhA monomer, while the integration method assigned a weight coefficient of less than 1 to the concentration of each RhA monomer. In particular, the plasma concentrations of the five RhA monomers were directly added numerically to obtain the plasma concentration of the total RhA using the adding-up method, so that the contribution of RhA monomers with low blood concentration was even ignored, while that with high-concentration monomers was highlighted. If the plasma concentration of each monomer was multiplied by the weight coefficient deduced by the bioavailability of RhA monomers, the difference in plasma concentration of different monomers can be reduced appropriately. Thus, the plasma concentration of total RhA can be calculated reasonably, and the contribution of RhA monomers with lower or higher plasma concentration to pharmacokinetic parameters may be reflected. As it is shown in Table 4, the integration method decreased $\mathrm{AUC}_{0-\infty}$ from $8584.88 \mathrm{ng} /$ $\mathrm{mL} \cdot \mathrm{h}$ to $3101.52 \mathrm{ng} / \mathrm{mL} \cdot \mathrm{h}$ and $\mathrm{C}_{\max }$ from 409.98 to $153.20 \mathrm{ng} / \mathrm{mL}$. The lower plasma concentration of the total rhubarb anthraquinones obtained by the integration method may indicate that a higher concentration of the non-absorbed rhubarb anthraquinones in the gastrointestinal tract may mainly produce a purgative effect after oral administration. We have to carefully suggest that this explanation was only for the possible synergistic purgative effect of rhubarb-free anthraquinones.

The plasma concentrations over time profiles of five RhA monomers can be fitted by different compartment models based on the fitting degree of R-squared, resulting in one more factor of error in pharmacokinetic parameters. Therefore, the classical pharmacokinetic compartment model was unfit for comparative pharmacokinetics study. In this case, a statistical moment model was chosen to analyze pharmacokinetic parameters. The concentration-related pharmacokinetic parameters like $\mathrm{C}_{\max }, \mathrm{AUC}_{0-\mathrm{t}}$, and $\mathrm{AUC}_{0-\infty}$ obtained by the addingup method were about 2-3 times higher than those obtained by the integration method, while the values of time-related pharmacokinetic parameters, such as MRT $_{0-t}$, MRT $_{0-\infty}$, Tmax and $t_{1 / 2 z}$, were in an indistinctive range between the two methods because their values were independent of the weighted sum of the plasma concentration of different monomers.
Plant drugs are generally composed of active substance groups, which exert their effects through multi-target and multi-pathway. However, the overall biological effects reflected by multiple components cannot be simply added based on the bioavailability and pharmacological action of each component. The interaction and connection between chemical components would affect their processes of absorption, distribution, metabolism, and excretion, and further, affect the holistic biological effect. Relatively, the integration method can offer a more thorough strategy by balancing the different pharmacokinetic properties among multiple components and the results might better explain the certain pharmacological effects (17). It is worth noting that the integration method can not completely explain the synergic effect of natural compounds, and it is only one of the possible ideas facing this issue. The main limitations of this study were: (1) There are no pharmacological effect data to support pharmacokinetic parameters; (2) It is difficult to clarify that pharmacological effect is mainly caused by rhubarb anthraquinones while not included other components. This question is the common problem faced by TCM research; (3) It should not be used to define a dosing scheme for patients before we can not scientifically establish the correlations between pharmacokinetic parameters and specific indications, e.g., purgative efficacy of rhubarb-free anthraquinones.

A rapid, simple, sensitive, and reliable HPLCFLD method was established and validated for simultaneous detection of rhubarb anthraquinones in rat plasma, and it was successfully applied to comparative pharmacokinetic study with adding-up and integrated plasma drug concentration calculation methods. The integrated pharmacokinetics of multiple components obtained with integrated plasma drug concentrations may help to better understand the certain biological efficacy in clinical practice.

\section{Conflicts of interest}

The authors declare that there are no conflicts of interest.

\section{REFERENCES}

1. Chang J.H., Zhang L., Gu X.D., Liu L.Y., Li Z.S., et al.: Drug Deliv. Sci. Technol. 43, 415 (2018).

2. Chen Y.K., Xu Y.K., Zhang H., Yin J.T., Fan X., et al.: Biomed. Pharmacother. 84, 1001 (2016).

3. Agarwal S.K., Singh S.S., Verma S., Kumar S.: J. Ethnopharmacol. 72, 43 (2000). 
4. Xiong H.R., Luo J., Hou W., Xiao H., Yang Z.Q.: J. Ethnopharmacol. 133, 718 (2011).

5. Iizuka A., Iijima O.T., Kondo K., Itakura H., Yoshie F., et al.: J. Ethnopharmacol. 91, 89 (2004).

6. Zhuang T.X., Gu X., Zhou N., Ding L.L., Yang L., et al.: Biomed. Pharmacother. 127, 110224 (2020).

7. Chen Y.X., Mei X.Q., Gan D.H., Wu Z.G., Cao Y.Q., et al.: Biomed. Pharmacother. 107, 226 (2018).

8. Chen Y.Y., Cao Y.J., Tang Y.P., Yue S.J., Duan J.A.: Chin. J. Nat. Med. 17, 871 (2019).

9. Zheng Y.F., Liu C.F., Lai W.F., Xiang Q., Li Z.F., et al.: Fitoterapia 96, 25 (2014).

10. Li J.C., Shi Y.B., Ren Y., Cong Z.T., Wu G.T., et al.: J. Drug Deliv. Sci. Technol. 39, 283 (2017).

11. Bali V., Ali M., Ali J.: Int. J. Pharm. 403, 46 (2011).
12. Merisko-Liversidge E., Liversidge G.G.: Adv. Drug. Deliv. Rev. 63, 427 (2011).

13. Shi Y.B., Li J.C., Ren Y., Wang H.Q., Cong Z.T., et al.: J. Drug. Deliv. Sci. Technol. 30, 242 (2015).

14. Wu W.J., Yan R., Li T., Li Y.P., Zhou R.N., et al.: J. Ethnopharmacol. 198, 600 (2017).

15. Zhang J., Fu Y.D., Li L., Liu Y., Zhang C., et al.: J. Pharm. Biomed. Anal. 171, 43 (2019).

16. Xiong F., Wang H., Jiang Z.Z., Huo M.R., Yan C.Y., et al.: J. Ethnopharmacol. 163, 290 (2015).

17. Zheng H.R., Chu Y., Zhou D.Z., Ju A.C., Li W., et al.: J. Chromatogr. B Analyt. Technol. Biomed. Life Sci. 1072, 282 (2018).

18. Wu W.J., Yan R., Yao M.C., Zhan Y., Wang Y.T.: Biomed. Chromatogr. 28, 564 (2014).

19. Zhang L., Chang J.H., Zhang B.Q., Liu X.G., Liu P., et al.: Fitoterapia 104, 86 (2015). 